\title{
Identification and Characterization of Genes Involved in the Fruit Color Development of European Plum
}

\author{
Dineshkumar Selvaraj, Sherif Sherif ${ }^{1}$, Mohd Sabri Pak Dek ${ }^{2}$, and Gopinadhan Paliyath \\ Department of Plant Agriculture, University of Guelph, Guelph, ON N1G2W1, Canada
Islam El-Sharkawy ${ }^{1}$ and Jayasankar Subramanian ${ }^{3}$
Department of Plant Agriculture, University of Guelph, 4890 Victoria Avenue North, P.O. Box 7000, Vineland Station, Vineland, ON LOR 2EO, Canada

\begin{abstract}
Additional index words. Prunus domestica, anthocyanin biosynthetic genes, polyphenols, chalcone synthase
Abstract. European plum fruit (Prunus domestica) are normally blue-black to dark purple. However, some genotypes remain green/yellow after ripening. We hypothesized that in such genotypes anthocyanin biosynthesis is genetically disturbed. To examine this hypothesis, six european plum genotypes with diverse fruit colors were investigated for the expression pattern of several anthocyanin biosynthetic genes (ABGs)—e.g., phenylalanine ammonia-lyase, chalcone synthase (CHS), dihydroflavonol 4-reductase (DFR), anthocyanin synthase (ANS), and UDP-glucose:flavonoid 3-Oglucosyltransferase 1 and 2 (UFGT 1 and 2). Expression profiles indicated that ABGs, especially $P d-C H S$ and $U F G T 2$, were significantly downregulated in the green/yellow fruit compared with the dark-purple fruit. Furthermore, the quantification of total polyphenols and individual flavonoid compounds showed substantial differences between the off-colored and the purple genotype. To further examine the contribution of each of the ABGs in color development, the open reading frame (ORP) of $P d-C H S, P d-D F R, P d-A N S$, and $P d-U F G T 2$ was ectopically expressed in tobacco (Nicotiana tabacum). The characterization of transgenic plants showed that the petals of plants expressing $P d-C H S$ were darker in color and had higher anthocyanin content than control or even other transgenic types, suggesting the significant contribution of CHS in determining anthocyanin production levels and hence fruit coloration. The results of this study provides better understanding of color development in european plum, which can be rewarding in developing european plum cultivars with desired colors through classical or modern breeding tools.
\end{abstract}

Consuming functional foods such as fruit and vegetables are important for reducing the risk of chronic diseases and maintaining good health. At least five to nine daily servings of fruits and vegetables are recommended by the U.S. Department of Agriculture (Zepeda et al., 2014). Fruit and vegetables provide essential vitamins, minerals, fibers (soluble and insoluble forms) as well as bioactive compounds collectively known as phytochemicals. These include polyphenols, carotenoids, and sulfur-containing components. In addition to health benefits, diversity in fruit colors is often associated with unique phytochemical constituents (Camara et al., 1995; Gould et al., 2010; Tzulker et al., 2007). Among several fruit in the Rosaceae family, european plum provide enormous amount of phytonutrients due to their high polyphenolic and fiber content thus constituting a functional health food.

Fruit color is a primary attribute to the appearance and quality of european plum. In general, color is important in

Received for publication 5 Nov. 2015. Accepted for publication 15 June 2016. This work was supported by grants from Ontario Ministry of Agriculture, Food and Rural Affairs (Canada) and Ontario Tender Fruit Producers Marketing board (OTFPMB).

We would like to thank K.S. Tiwari, C.M. Ajila, and Renu Chandrasekaran (University of Guelph, ON, Canada) for their technical support during the research work.

${ }^{1}$ Current address: Department of Horticulture, Faculty of Agriculture, Damanhour University, Al-Gomhuria Street, P.O. Box 22516, Damanhour, Al-Behira, Egypt.

${ }^{2}$ Current address: Department of Food Science, Faculty of Food Science and Technology, Universiti Putra Malaysia, 43400 Serdang, Selangor, Malaysia.

${ }^{3}$ Corresponding author. E-mail: jsubrama@uoguelph.ca. attraction of dispersal agents (birds, animals, and primates), protection against ultraviolet damage, an indicator of ripeness, and contributes to polyphenolic content and their associated antioxidant properties (Davies and Schwinn, 2004). As the fruit ripens, color is one of many modifications that occur due to physiological and biochemical changes; including the increase in respiration rate, production of ethylene, flesh softening, and formation of volatiles with associated development of flavor. During the ripening of european plum, there is loss of chlorophyll pigment that leads to development of blue-black, purple, or yellow pigmentation (Abdi et al., 1998). In higher plants these pigments are localized in chromoplasts or vacuoles (Kahlau and Bock, 2008). The compounds responsible for this diverse pigmentation are anthocyanins synthesized through a specific biosynthetic pathway in most plants, including european plum.

Anthocyanin pathway includes an early biosynthetic pathway that leads to the production of colorless dihydroflavonol compounds and late biosynthetic pathway that leads to the production of anthocyanins (color pigmentation) (Holton and Cornish, 1995; Jaakola et al., 2002). The activity of ABGs is believed to be crucial in the formation of anthocyanins with different structure and coloration including conjugates of cyanidin (red), pelargonidin (orange), and delphindin (blue) (Dooner et al., 1991). The expression of the ABGs depends on several factors; including tissue, genotype, developmental stage, and environment (Boss et al., 1996; Takos et al., 2006); and may serve as qualitative and quantitative indicator of pigmentation in ripe fruit. Molecular mechanisms underlying 
the regulation of anthocyanin biosynthetic pathway have been investigated in some species, such as grape (Vitis vinifera), bilberry (Vaccinium myrtillus), and malay apple (Syzygium malaccense), but not well understood in the case of european plum (Fournand et al., 2006; Jaakola et al., 2002; Kotepong et al., 2011).

In the present study, european plum genotypes with varied fruit colors ranging from green to purple were used as a model system to better understand the role of anthocyanin biosynthetic pathway in the regulation of european plum pigmentation at ripening. The expression of key ABGs was assessed during european plum fruit ripening and the functional analysis of individual $\mathrm{ABG}$ was examined using tobacco as a heterologous experimental system. The findings of this research can be used in breeding programs aiming at obtaining european plum with desired colors for the market and consumers.

\section{Materials and Methods}

Plant materials. European plum with accession numbers V982017 (purple), V90271 (greenish purple), V91057 (yellow 1), V91058 (yellow 2), V91048 (green 1), and V95141 (green 2) grown at Vineland Research and Innovation Center farm, Vineland Station, ON, Canada, were used in this study (Fig. 1). Fruit were collected at the commercial harvest stage and fruit skin was peeled off. Peel samples were frozen immediately in liquid nitrogen and then stored at $-80{ }^{\circ} \mathrm{C}$ until further analysis.

Gene EXPRESSION ANALYSES. Total RNA from peel samples was extracted using a modified cetyltrimethylammonium bromide protocol (Meisel et al., 2005). RNA samples were treated with DNase I (Thermo Fisher Scientific, Mississauga, ON, Canada) before the synthesis of complementary DNA (cDNA)
A
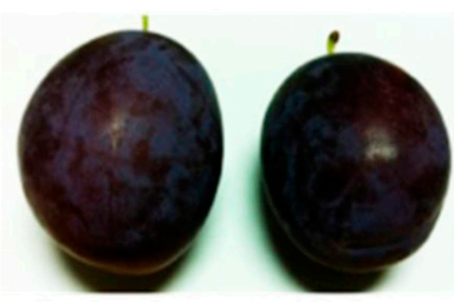

C

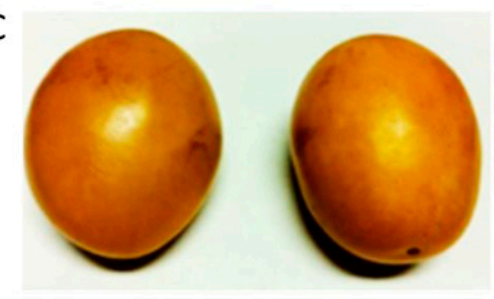

$\mathrm{E}$

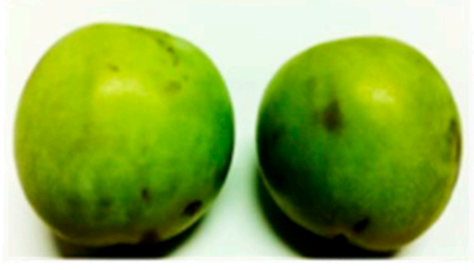

$\mathrm{F}$

D
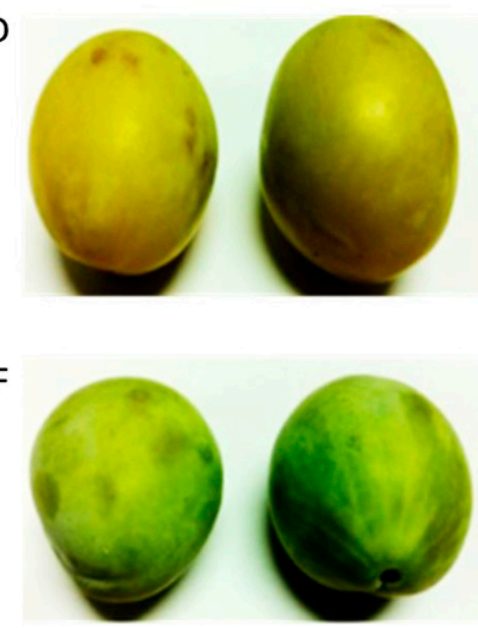

Fig. 1. Novel genotypes of european plum examined in this study. Genotypes (A) purple (V982017), (B) greenish purple (V90271), (C) yellow 1 (V91058), (D) yellow 2 (V91057), (E) green 1 (V91048), and (F) green 2 (V95141). to remove any traces of genomic DNA. To examine the efficacy of DNase treatment, treated RNA samples were examined by PCR using Pd-Actin primers (Supplemental Table 1). No amplification was observed in DNase-treated RNA samples (data not shown), indicating the absence of genomic DNA in these samples. The first-strand cDNA was synthesized from $5-\mu \mathrm{g}$ of DNase-treated RNA using Revert Aid ${ }^{\mathrm{TM}} \mathrm{H}-$ Minus First Strand cDNA Synthesis Kit (Thermo Fisher Scientific) according to manufacturer's instructions. Quantitative real-time polymerase chain reaction (qRT-PCR) was performed using real-time PCR detection system (CFX Connect ${ }^{\mathrm{TM}}$; Bio-Rad Laboratories, Mississauga, ON, Canada). Primers were designed for qRT-PCR, using Primer3Plus software [Untergasser et al., 2007 (Supplemental Table 2)]. PCR reactions were carried out in a $25-\mu \mathrm{L}$ reaction mixture containing $\mathrm{iQ}^{\mathrm{TM}} \mathrm{SYBR}$ Green Supermix (BioRad Laboratories), $0.2 \mu \mathrm{M}$ of each primer and $100 \mathrm{ng}$ of cDNA. The threshold cycle (CT) values for each gene were normalized to plum $\beta$-actin $(P d$-Actin). Expression of each gene was calculated as $2^{-\Delta \mathrm{CT}}$ where $\Delta \mathrm{CT}=\mathrm{CT}_{\text {target gene }}-\mathrm{CT}_{P d \text {-Actin. The }}$ relative normalized gene expression was calculated as $2^{-\Delta \Delta \mathrm{CT}}$ $\left(\Delta \Delta \mathrm{CT}=\Delta \mathrm{CT}_{\text {treatment }}-\Delta \mathrm{CT}_{\text {control }}\right)$. The purple genotypes were always considered as control (calibrator) samples.

Isolation of ABGs. Full-length sequences of five ABGs, designated $P d-P A L, P d-C H S, P d-D F R, P d-A N S$, and $P d-U F G T$ (GenBank accession numbers are listed in Supplemental Table 2) were isolated from frozen fruit skin tissues (at the commercial harvesting date) using the reverse transcription PCR (RTPCR) approach. Forward and reverse primers flanking the coding region of these genes were designed based on peach (Prunus persica) sequences. Purified PCR products were sequenced using 3730 DNA Analyzer (Life Technologies, Burlington, ON, Canada) and deposited in National Center for Biotechnology Information. Primers used for ABGs isolation are listed in Supplemental Table 3.

QUANTIFICATION OF TOTAL POLYPHENOLS IN EUROPEAN PLUMS. European plum fruit peel from 10 fruit was homogenized in $100 \%$ methanol using a homogenizer fitted with a probe (Polytron PTA 10; Brinkmann Instruments, Mississauga, ON, Canada). The homogenate was centrifuged at $1600 g_{n}$ for $15 \mathrm{~min}$. The supernatant was collected and aliquots were stored at $-80{ }^{\circ} \mathrm{C}$ for further analysis. The extracts were initially evaporated in a Rotovapor R (Buchi Labortechnik, Meierseggstrasse, Switzerland) to remove methanol and residual methanol was dried in a stream of nitrogen gas. The dried residue was resuspended in distilled water and loaded on to a C18 Sep-Pak cartridge (Waters Corp., Milford, MA). Total crude polyphenol fractions were eluted with $100 \%$ methanol. The levels of total polyphenols were estimated using the modified Folin-Ciocalteau method (Waterhouse, 2002) and quantified spectrophotometrically at $765 \mathrm{~nm}$. The total polyphenol concentration was expressed as weight of gallic acid equivalent/100 $\mathrm{g}$ fresh weight.

Chromatographic analyses of crude european plum fractions (polyphenolic extracts) 
were performed on an ultra-high-performance liquid chromatography. Ultimate 3000 liquid chromatograph interfaced to an amazon SL ion trap mass spectrometer (Dionex; Bruker Daltonics, Billerica, MA) in both positive and negative ion modes. A C18 high-performance liquid chromatography (HPLC) column [2.7- $\mu \mathrm{m}$ particle size, $150 \times 4.6 \mathrm{~mm}$ (Poroshell 120 EC18; Agilent Technologies, Mississauga, ON, Canada)] was used for chromatographic separation. Elution was performed using mobile phase A (water:formic acid, 99.9:0.1 v/v) and mobile phase B (acetonitrile:formic acid, 99.9:0.1 v/v) at a flow rate was $0.4 \mathrm{~mL} \cdot \mathrm{min}^{-1}$ and the compounds detected at 260 and $520 \mathrm{~nm}$. The linear gradient used was as follows: 0 $2 \mathrm{~min}, 93 \% \mathrm{~A}$ and $7 \% \mathrm{~B}$; 2-33 min, 20\% B; 33-41 min, 80\% B; 41-46 min, 100\% B. Nitrogen was used as both nebulizing and drying gas, helium was used as collision gas. Electrospray ionization (ESI) conditions were as follows: nitrogen pressure: $275.8 \mathrm{kPa}$; helium pressure: $413.7 \mathrm{kPa}$; drying gas $10 \mathrm{~L} \cdot \mathrm{min}^{-1}$ at $250{ }^{\circ} \mathrm{C}$; electrospray capillary voltage $4.5 \mathrm{~V}$. The mass-tocharge ratio scanning range was from 100 to $2200 \mathrm{~m} / \mathrm{z}$ in positive ion enhanced resolution mode. Auto tandem mass spectrometry (MS) fragmentation mode was selected for the first two ions with the highest intensity. A fragmentation amplitude of 1 was applied with smart parameter setting mode, which automatically optimizes the trap drive level for precursor ions. The instrument was externally calibrated with the ESI TuneMix (Agilent Technologies). An appropriate aliquot of the samples, usually $20 \mu \mathrm{L}$ (40 $\mu \mathrm{g}$ polyphenols) was injected for the analysis.

GENETIC TRANSFORMATION OF TOBACCO. The ORFs of $P d$ $C H S, P d-D F R, P d-A N S$, and $P d-U F G T 2$ were amplified from european plum cDNA library, using the primers listed in Supplemental Table 4. The amplified fragments were then digested with $B a m \mathrm{HI}$ and cloned into the pGreen binary vector (Hellens et al., 2000) in-frame with the N-terminal of GFP and upstream the CaMV dual $35 \mathrm{~S}$ promoter. Plasmids containing the designated genes were then introduced to Agrobacterium tumefaciens C58 (harbouring pSoup plasmid) by electroporation. Tobacco cv. PetH4 plants were transformed using leafdisc method (Horsch et al., 1985).

QUANTIFICATION OF TOTAL ANTHOCYANIN IN TRANSGENIC FLOWERs. Anthocyanins were extracted from five disks $(15 \mathrm{~mm}$ diameter) of control and transgenic tobacco flowers ectopically expressing $P d-C H S, P d-D F R, P d-A N S$, and $P d-U F G T 2$ by soaking overnight with isolation solution $(2.7 \mathrm{~mL}$ methanol and $300 \mu \mathrm{L}$ of $1 \mathrm{~N} \mathrm{HCl}$ ) (Sigma-Aldrich, Oakville, ON, Canada) at $4{ }^{\circ} \mathrm{C}$. Anthocyanin extracts were filtered through glass wool and 0.45- $\mu \mathrm{m}$ Millex-HV filter (Thermo Fisher Scientific) before total quantification at $\mathrm{A}_{520} \mathrm{~nm}$ using a spectrophotometer. The total anthocyanin content was expressed as weight of cyanidin equivalent [extinction coefficient $(\varepsilon): 26,900 \mathrm{~L} \cdot \mathrm{cm}^{-1} \cdot \mathrm{mol}^{-1}$ ] and micrograms of anthocyanins per gram fresh weight was calculated according to the following Beer-Lambert formulae, A $\varepsilon$ CL, where $\mathrm{A}$ is the absorbance (no units); $\mathrm{C}$ is the concentration in moles per liter; $\mathrm{L}$ is the path length of the cuvette, expressed in centimeters.

The purified anthocyanin extracts $(20 \mu \mathrm{L}$ injections) were analyzed by HPLC (e2695 Alliance with ultraviolet/Visible detector, Waters) on a $3.0 \times 150 \mathrm{~mm}$ column of $5 \mu \mathrm{m} \mathrm{C18}$ (XTerra Mass Spectrometry, Waters) using a 22-min isocratic method and eluted with acetonitrile:water $(50: 50 \mathrm{v} / \mathrm{v})$ at $0.4 \mathrm{~mL} \cdot \mathrm{min}^{-1}$. Eluted compounds were detected at $520 \mathrm{~nm}$. The amount of anthocyanin was compared against authentic standards, cyanidin-3-glucoside (Sigma-Aldrich).
Statistical analysis. One-way analysis of variance (ANOVA) using proc generalized linear model procedures was performed using SAS (version 9.2; SAS Institute, Cary, NC). All parameters were examined for normality before ANOVA and $\log$ transformation was carried out when required. For gene expression, $2^{-\Delta \Delta \mathrm{CT}}$ (values of relative gene expression) was used for ANOVA and means comparison. Tukey's test was used to compare among the means. The data are presented as means \pm SE of means. The different alphabets in the figures indicate significant difference at $P<0.05$.

\section{Results}

TOTAL POLYPHENOL CONTENT IN SIX GENOTYPES OF EUROPEAN PLUM. The relative amounts of total polyphenolic compounds present in the six genotypes of european plum were analyzed and presented in Fig. 2 and Supplemental Table 5. Purple plums were used as a standard for comparison. Although there was no significant difference between the two yellow genotypes based on their total polyphenol content, yellow 1 showed a significantly less amount of polyphenols than purple plums. The two green genotypes showed a $70 \%$ reduction in total polyphenol contents relative to the standard, purple genotypes.

ANALYSIS OF SPECIFIC POLYPhenOLs. Using LC-MS, phenolic compounds, phenolic acids, flavonoids, and anthocyanins were detected in the six european plum genotypes (Supplemental Tables 6-11). Among phenolic acids, quinic acid, chlorogenic acid, and diferulic acid were present in all genotypes. Malic acid, dihydroferulic acid, and caffeyl tartaric acid were identified, but only in greenish-purple and green 2 genotypes. Some phenolic glucosides, such as dihydroferulic acid glucosides and caffeic acid glucosides were distinctly abundant in green and purple genotypes. However, the levels of total phenolic acids were different among the genotypes. Relative to the purple genotype, all investigated european plum genotypes exhibited significantly lower total phenolic acids content (Fig. 3A). Among the flavonoids, quinochalcone and kaempferol were identified in all genotypes. Quercetin derivatives were detected only in greenishpurple and the two green genotypes, whereas chalcone was identified only in yellow genotypes. Amounts of total flavonoids were also different among genotypes.

A reduction in total flavonoids was observed in the greenish purple, yellow 1, yellow 2, green 1, and green 2 compared with

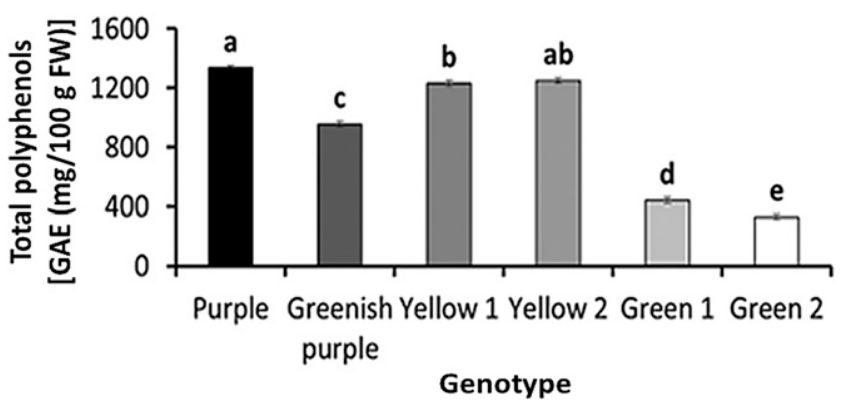

Fig. 2. Total polyphenol content expressed as gallic acid equivalents in different genotypes of european plum. The bars represent the mean \pm SE of three biological replicates ( $\mathrm{n}=10$ fruit). Values marked with same letters are not significantly different at $P<0.05$ by one-way analysis of variance followed by post hoc Tukey's test. 


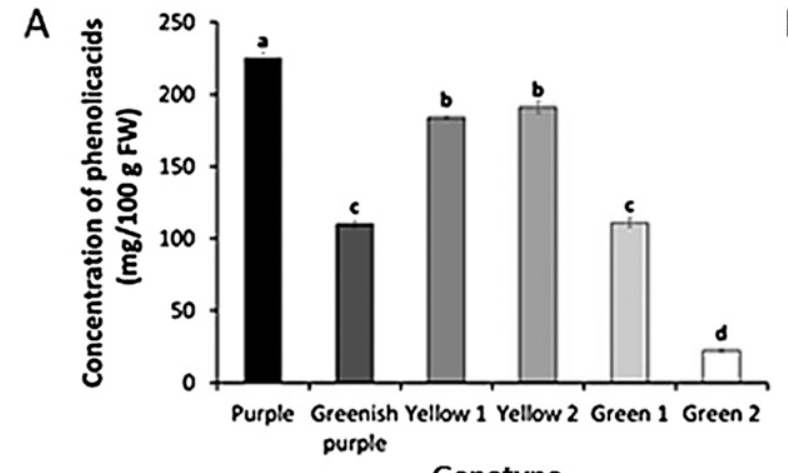

Genotype

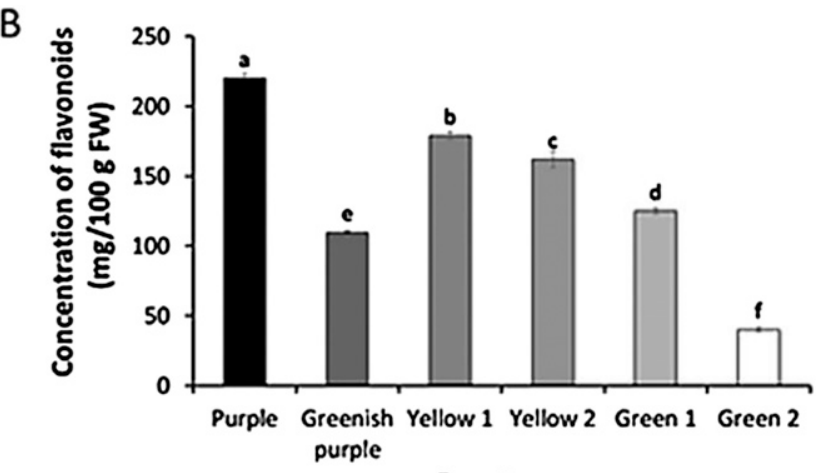

Genotype

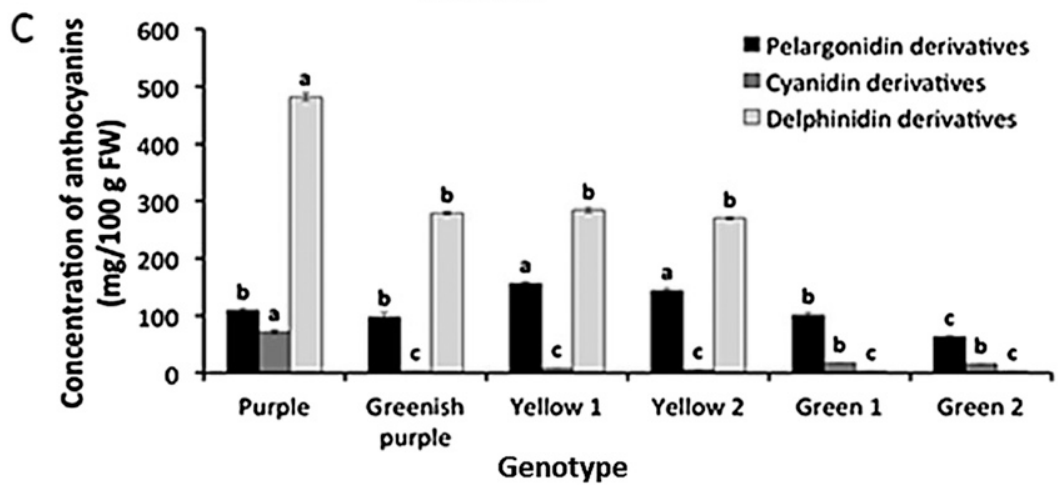

Fig. 3. Amount of individual (A) phenolic acids, (B) flavonoids, and (C) anthocyanin derivatives in different genotypes of european plum. Anthocyanin derivatives such as delphinidin, cyanidin, and pelargonidin are present in all genotypes but with varying concentrations. Delphinidin derivatives are present in very low amounts in green genotypes compared with yellow and purple genotypes. The bars represent the mean \pm SE of three biological replicates ( $\mathrm{n}=10$ fruit). Values marked with same letters are not significantly different at $P<0.05$ by one-way analysis of variance followed by post hoc Tukey's test.

the purple genotype (Fig. 3B). Interestingly, the flavonoid content showed more abundance than the phenolic acids in the case of green genotypes (Fig. 3A and B). Among the anthocyanins, pelargonidin, cyanidin, and delphinidin were identified in all genotypes. Distinct compounds, such as malvidin 3-O-glucoside (derivative of delphinidin) and monardaein 3-O-glucoside (derivative of pelargonidin) were identified in greenish-purple and green european plums, but not in yellow and purple european plums.

A reduction in total anthocyanin content (compared with purple genotypes) was observed in all genotypes particularly more in both green genotypes (Fig. 3C). The percentage of reduction in total anthocyanin content are as follows: $41.3 \%$, $32.3 \%, 36.6 \%, 81.7 \%$, and $87.6 \%$ in greenish-purple, yellow 1 , yellow 2, green 1, and green 2 genotypes, respectively. Among the anthocyanin derivatives, delphinidin was present in low amounts while pelargonidin was detected in high amounts in both green genotypes compared with other genotypes. Among the total anthocyanin derivatives, delphinidin derivatives were found to be $72.6 \%, 71.5 \%, 63.2 \%$, and $64.3 \%$ in purple, greenish-purple, yellow 1, and yellow 2 genotypes, respectively; whereas only $0.02 \%$ and $0.03 \%$ of these compounds were detected in green 1 and green 2 genotypes, respectively (Supplemental Tables 6-11).

Among the total anthocyanin derivatives, pelargonidin derivatives were found to be $16.4 \%, 25.1 \%, 34.7 \%$, and $34.2 \%$ in purple, greenish-purple, yellow 1, and yellow 2 genotypes, respectively. However, these were much higher in green 1 and green 2 genotypes with amounts of $83.4 \%$ and $78.9 \%$, respectively. Among the total anthocyanin derivatives, cyanidin derivatives were found to be $10.8 \%, 14.2 \%$, and $18.6 \%$ in purple, green 1, and green 2 genotypes, respectively; and very low in greenish-purple, yellow 1, and yellow 2 genotypes with amounts ranging $2.7 \%, 8.2 \%$, and $5.4 \%$, respectively. LC-MS profiles of polyphenols from six genotypes are listed in Supplemental Tables 6-11.

GENE EXPRESSION ANALYSIS OF ANTHOCYANIN BIOSYNTHESIS GENES. To identify genes involved in the metabolic flux toward the anthocyanin production, the expression patterns of ABGs namely PAL, CHS, DFR, ANS, UFGT 1, and UFGT 2 were assessed in the six diversely pigmented genotypes of mature european plum. With the exception of UFGT 1, the expression of all genes was significantly downregulated in the greenishpurple, yellow, and green fruit relative to purple fruit. The transcripts of $P d-P A L$ decreased by $50 \%$ in greenish purple, $70 \%$ in green $1,90 \%$ in green 2 , and more than $90 \%$ in the two yellow genotypes (Fig. 4A). Similarly, transcripts of $\mathrm{Pd}$-CHS were downregulated in all the five genotypes compared with the purple (Fig. 4B). The expression of $P d-D F R$ decreased $70 \%$ in green 1, but was notably downregulated in greenish-purple, and the two yellows and green 2 european plums (Fig. 4C). The level of ANS transcript was reduced by $70 \%$ in both yellow and green 1 genotypes; whereas, a reduction of $90 \%$ was apparent in the green 2 genotype (Fig. 4D). For the UFGT, the expression profile was different between the two copies identified for this gene in european plum, $P d-U F G T 1$ and $P d$-UFGT 2. Interestingly, there is a $10 \%$ and $60 \%$ increase in $P d$-UFGT 1 in the yellow 2 and green 1 genotypes, respectively; however, its levels decreased to $40 \%, 70 \%$, and $50 \%$ in greenish-purple, yellow 1, and green 2 genotypes, respectively 
A

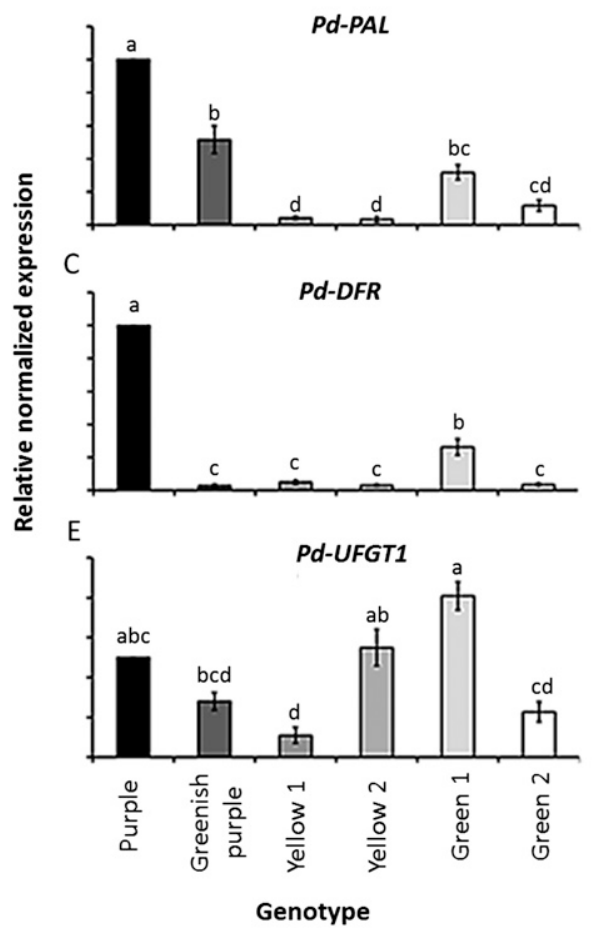

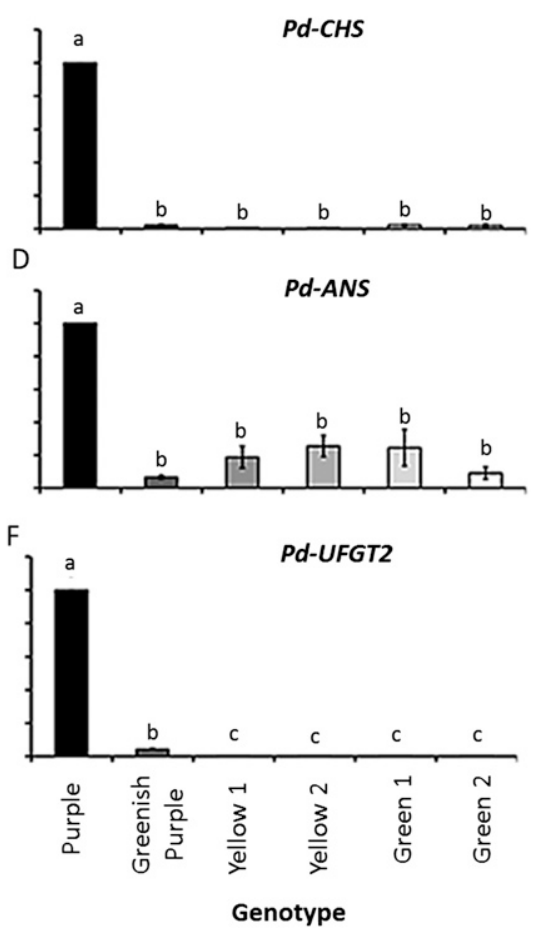

Fig. 4. Gene expression analysis of (A) phenylalanine ammonia-lyase (PAL), (B) chalcone synthase (CHS), (C) dihydroflavonol reductase (DFR), (D) anthocyanin synthase (ANS), (E) UDP-glucose flavanoid 3-O glucosyl transferase ( $U F G T$ 1), and (F) UDP-glucose flavanoid 3-O glucosyl transferase (UFGT 2) in six genotypes of european plum. The expression of each gene was normalized to that of $P d$-Actin and was calculated relative to the expression in the purple genotype (calibrator). The values are mean \pm SE of three biological replicates. Values marked with same letters are not significantly different at $P<0.05$ by one-way analysis of variance followed by post hoc Tukey's test.

(Fig. 4E). By contrast, the expression for UFGT 2 was significantly downregulated in all genotypes compared with the purple genotype (Fig. 4F).

The ECTOPIC EXPRESSION OF ABGs IN TOBaCCo. Transgenic tobacco plants ectopically expressing $P d-C H S, P d-D F R, P d-$ $A N S$, and $P d-U F G T$ had vegetative growth and fertility similar to their wild-type (WT) counterparts. However, there were unique differences in petal color intensity between the flowers of WT and transgenic plants expressing $P d-C H S\left(\mathrm{CHS}_{\exp }\right), P d-D F R$ $\left(\mathrm{DFR}_{\text {exp }}\right), P d-A N S\left(\mathrm{ANS}_{\mathrm{exp}}\right.$ ), and $P d-U F G T 2$ (UFGT ${ }_{\text {exp }}$ ) (Fig. $5 \mathrm{~A})$. On the other hand, petals of WT and positive control plants (expressing the pGreen binary vector only) showed similar color intensities. The petals of $\mathrm{CHS}_{\exp }$ were dark pink in color after complete emergence and remained dark and highly pigmented throughout flower development. The petals of DFR $\mathrm{exp}_{\mathrm{e}}$ were very pale pink in color and remained less pigmented than control flowers through growth. However, visual inspection of the petals of $\mathrm{ANS}_{\text {exp }}$ and $\mathrm{UFGT}_{\text {exp }}$ revealed similar color intensity to that of control flowers. All the five transgenic lines of each transgene showed similar color pattern.

The QUANTIFICATION OF TOTAL ANTHOCYANIN IN TRANSGENIC PLANTS. Anthocyanin content was quantified in petals of control as well as transgenic tobacco plants. Although WT and positive control plants were similar in anthocyanin content, anthocyanins were found to be higher in petals of CHS $_{\exp }(0.40 \mu \mathrm{g})$ compared with control tobacco plants. On the other hand, the concentration of anthocyanins were significantly lower in the petals of $\mathrm{DFR}_{\exp }(0.08 \mu \mathrm{g})$ compared with control plants (Fig. 6B). The anthocyanin levels in petals of $\mathrm{UFGT}_{\exp }, \mathrm{ANS}_{\exp }$ were not significantly different compared with control. Cyanidin derivatives (the only anthocyanin found in the tobacco petals) were taken as an equivalent for calculating the concentration.

\section{Discussion}

To study the function of CHS, DFR, ANS, and UFGT enzymes in the anthocyanin biosynthetic pathway, an efficient model system was required. Although transformation of plum with foreign genes has been described previously (Hily et al., 2005), the generation of a stably transformed european plum trees requires an extended period of time and fruiting on these trees will be even longer, at least 5 years. Although it may not be the best choice, tobacco was chosen as an experimental model system because of its relatively high homogeneity, regenerative ability, short life cycle, and, more importantly, its flowers in which modifications in anthocyanin content can be easily detected and quantified. Indeed, modification of flower color was accomplished in transgenic tobacco plants by the ectopic expression of two genes $P d-C H S$ and $P d-D F R$ from european plum that are involved in anthocyanin pathway. Result from this study strongly suggests that $P d$-CHS expression resulted in enhanced cyanidin glycoside biosynthesis, signifying its potential regulatory role in plum. It is likely that in plums the coloration is primarily regulated at the CHS level. With limiting amounts of naringenin flavanone, activities of other enzymes downstream may not be able to produce higher amounts of cyanidin glycoside that results in the red coloration of plums. Although in tobacco flowers the coloration of flowers in transgenic plants further confirmed the positive role of Pd-CHS, the nonsignificant role of downstream enzymes (e.g., Pd-DFR, Pd-ANS, and PdUFGT) in transgenic tobacco cannot discount their possible function in anthocyanin biosynthesis. Indeed, other factors such as the different substrate specificity between plum and tobacco and/or the natural deficiency of some products (e.g., pelargonidin) in tobacco (Nakatsuka et al., 2007) might be involved in the resulting phenotypes of flowers in transgenic plants.

Although the biosynthesis of anthocyanin in many plants is always associated with phenylalanine ammonia lyase (PAL) activity, there are many examples of high PAL activity without anthocyanin production (Jones, 1984; Lister et al., 1996). In essence, PAL is active in the biosynthesis of other phenylpropanoid compounds such as chlorogenic acid, coumarin, and lignin (Zhang and Liu, 2015), suggesting that enzymes downstream PAL might contribute to the different anthocyanin content among plum genotypes. Our results clearly indicate that Pd-CHS might be one of the critical enzymes involved in anthocyanin production in european plum fruit at ripening. High transcription levels of 
A

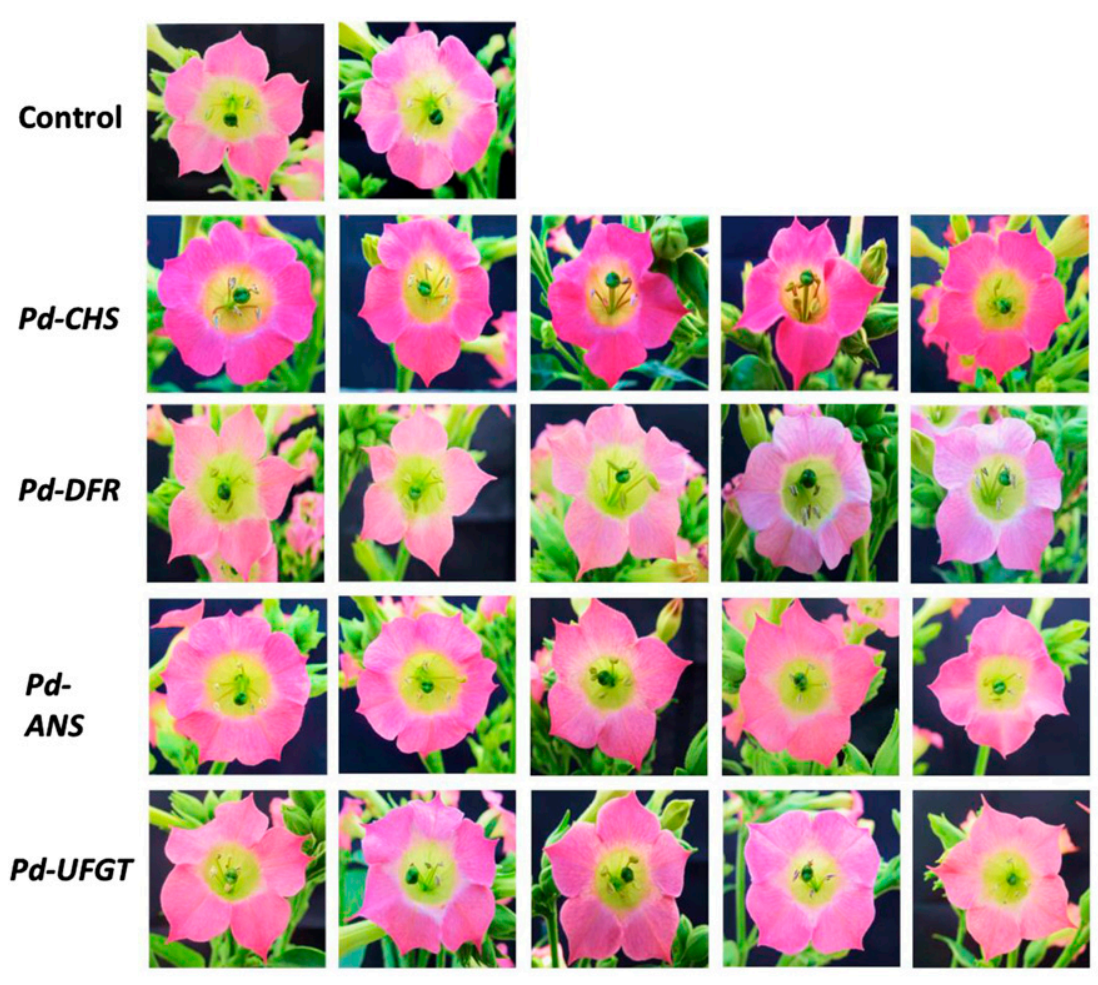

B

Phenyl alanine

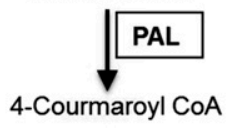

Naringenin chalcone

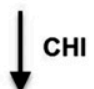

Naringenin flavanone

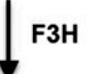

Dihydroflavonols
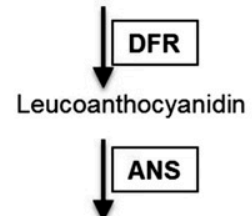

Anthocyanidin

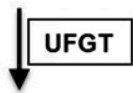

Anthocyanins

Cyanidin Pelargonidin Delphinidin glucoside glucoside glucoside

(red) (orange) (blue)

Fig. 5. Phenotypic characterization of control and transgenic tobacco flowers. (A) Flowers collected from five lines of transgenic plants expressing Prunus domestica chalcone synthase (Pd-CHS), $P$. domestica dihydroflavonol reductase (Pd-DFR), $P$. domestica anthocyanin synthase (Pd-ANS), and P. domestica UDP-glucose flavanoid 3-O-glucosyl transferase (Pd-UFGT) were evaluated based on corolla color intensity. (B) Schematic representation of anthocyanin biosynthetic pathway. All the enzymes involved in the pathway are in bold. The candidate genes examined in this study are boxed.

$P d$-CHS was only associated with intense purple color. Furthermore, the ectopic expression of $P d-C H S$ in tobacco converted the light pink petals of WT into dark pink petals. The quantification of anthocyanin in WT and $\mathrm{CHS}_{\text {exp }}$ petals showed significant differences supporting the dark pink phenotype of transgenic plants. Recent studies have indicated that expression of $\mathrm{CHS}$ is closely correlated with anthocyanin accumulation of blood-flesh peach (Jiao et al., 2014). In addition, the silencing of CHS in tomato (Solanum lycopersicum) fruit by virus-induced gene silencing has also shifted the characteristic orange color of tomato fruit into pink color due to the altered flavonoid content in the $\mathrm{CHS}$ gene-silenced fruit (Espana et al., 2014). It is noteworthy that the influence of each of the anthocyanin biosynthetic enzymes on fruit pigmentation is different among plant species. For instance, in apple (Malus sylvestris), the expression of most genes involved in the anthocyanin biosynthetic pathway is positively correlated with anthocyanin accumulation in fruit (Honda et al., 2002; Kim et al., 2003). However, in grape and european pear (Pyrus communis), only the action of UFGT enzyme is considered the key step in anthocyanin biosynthesis that determines the development of red coloration in these fruit (Boss et al., 1996; Kobayashi et al., 2004; Wang et al., 2013). Similarly, the glycosylation-related gene LcUFGT1 was found to play a critical role in the formation of the red color in the pericarp of lychee [Litchi chinensis (Li et al., 2015)].

Dihydroflavonol reductase is perhaps the subsequent limiting step next to CHS in the production of anthocyanins in the off-colored genotypes. The transcript levels of gene encoding dihydroflavonol reductase were significantly lower in green and yellow compared with purple, except green 1 genotype. Unexpectedly, the ectopic expression of $P d-D F R$ in tobacco resulted in pale pink-colored petals indicating the loss of anthocyanins, probably due to the inhibition mechanism. Also, the anthocyanin content in WT and $\mathrm{DFR}_{\text {exp }}$ petals reveal the significant differences explaining the pale pink colored petals. Current studies showed that DFR transcript levels were higher in red fruit bearing woodland strawberry (Fragaria vesca) compared with yellow fruit (Xu et al., 2014). Moreover, the silencing of $D F R$ in strawberry fruit by agro-filtration showed less intense color than normal fruit. Studies also indicate that overexpression of the cranberry (Vaccinium macrocarpon) $D F R$ could be used to increase anthocyanin production in transgenic plants (Polashock et al., 2002). These findings jointly suggest that the differential regulation of $D F R$ genes among different genotypes of european plum is responsible for differential anthocyanin synthesis between the green/yellow and the purple genotypes of european plum.

ANS is a key enzyme, believed to participate in the metabolic flux of anthocyanin and its derivatives in european plum fruit. The transcripts levels of $P d-A N S$ were considerably higher compared with transcripts of $P d-C H S$ and DFR in the off-colored genotypes. Moreover, the ectopic expression of $P d-A N S$ in tobacco turned the light pink-colored petals into pink-colored petals. The differential expression patterns of $A N S$ were decisive and led to color difference between european pear and asian pear (Pyrus pyrifolia) (Yang et al., 2015). Silencing of anthocyanidin synthase in redleaf apple cultivar resulted in blockage of anthocyanin synthesis 


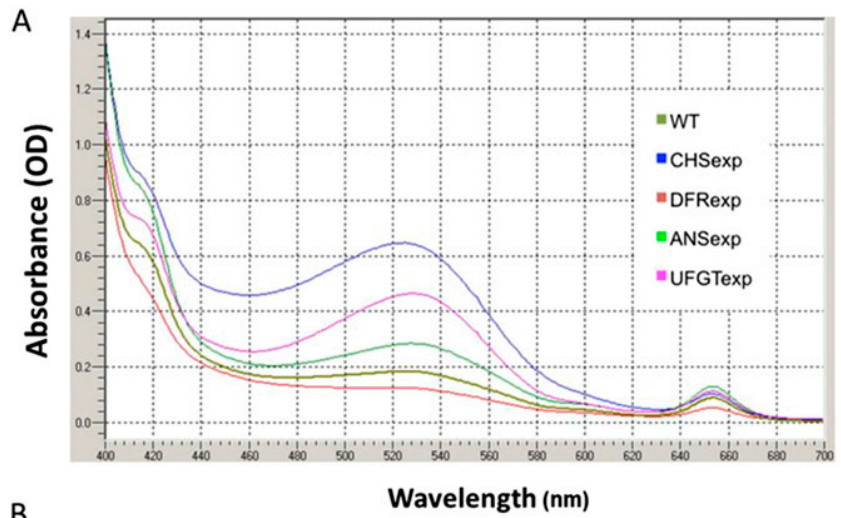

B

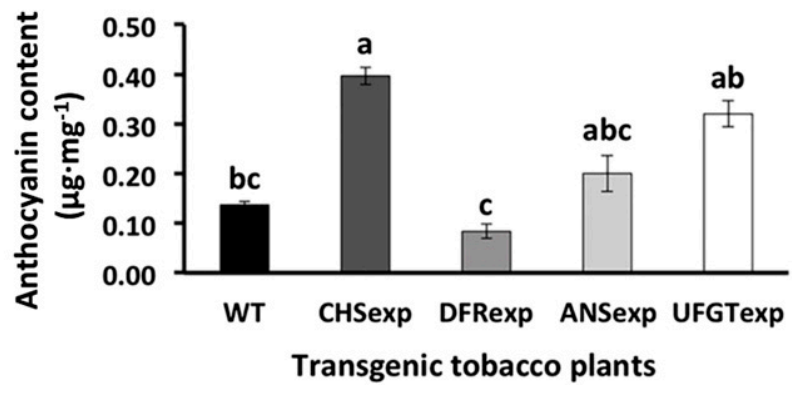

Fig. 6. Anthocyanin content in flowers of wild-type and transgenic tobacco plants. (A) Anthocyanin absorption spectrum at $520 \mathrm{~nm}$ (optical density) for wild-type and transgenic tobacco plants. (B) Anthocyanin content present in flowers of transgenic plants expressing Prunus domestica chalcone synthase (Pd-CHS), $P$. domestica dihydroflavonol reductase (Pd-DFR), $P$. domestica anthocyanin synthase (Pd-ANS), and $P$. domestica UDP-glucose flavanoid 3-O-glucosyl transferase (Pd-UFGT) along with wild-type (WT) tobacco plants. The concentration of anthocyanin content is calculated according to the extinction coefficient of cyanidin $26,900 \mathrm{~L} \cdot \mathrm{cm}^{-1} \cdot \mathrm{mol}^{-1}$. Values marked with same letters are not significantly different at $P<0.05$ by one-way analysis of variance followed by post hoc Tukey's test.

and accompanied by increase in flavonoids, polyphenols, and importantly epicatechin (Szankowski et al., 2009). These studies suggest that though anthocyanidin synthase is important for anthocyanin synthesis, the metabolic flux are different among systems and may be deviated to other pathways for synthesis of compounds like pronthocyanidins.

This was the first attempt to express the last downstream enzyme $U F G T$ from european plum. The transcripts levels of two isoforms of $U F G T$ were different among the green/yellow compared with purple genotype. The transcripts of UFGT 1 were relatively higher in some yellow and green genotypes compared with purple genotypes; whereas, the expression of $U F G T 2$ was downregulated in all off-colored genotypes. Furthermore, the ectopic expression of $P d-U F G T 2$ in tobacco resulted in pink-colored petals similar to $P d-A N S$. However, the quantification of anthocyanin content in the petals of these plants did not show any significant difference (Fig. 6B). Interestingly, transcript levels of UFGT are low in the color mutant Malay apple with white skin compared with the WT fruit (Kotepong et al., 2011). Studies reveal that when UF3GT from Freesia hybrida was introduced into Petunia grandiflora, there was a reduction in UFGT transcript levels and significant changes in flower color of petunia from red to pink (Sui et al., 2011). These findings suggest that these two forms of $U F G T$ in plums may act as a control point in the anthocyanin metabolic flux leading to different anthocyanin derivatives among the different genotypes of european plum. It is also worth noting that UFGT and other enzymes involved in the flavonoid biosynthesis (e.g., CHS) are encoded by multigene families (Durbin et al., 2000; Lim et al., 2003), which suggests that other UFGT paralogs might be expressed in different ratios among plum genotypes, resulting in different fruit colors at ripening.

The presence of three anthocyanins namely cyanidin glucoside, pelargonidin glucoside, and delphinidin glucoside in purple european plum indicates the substrate preference of the DFR toward all three dihydroflavonols (dihydroquercetin, DHQ; dihydrokaempferol, DHK; and dihydromyricetin, DHM). In purple genotypes, the anthocyanin metabolic flux is more toward DHM as the delphinidin-based derivatives are high, thus resulting in the purple-bluish color of the fruit. In greenish-purple and yellow genotypes, the flux is toward DHK and DHM as pelargonidin and delphinidin derivatives exist. Interestingly, green genotypes have flux only toward DHK, very less flux toward DHQ and no flux toward DHM. There are two possible mechanisms for the different anthocyanin metabolic flux in different genotypes of european plum, 1) the enzymes in the upstream anthocyanin biosynthetic pathway (CHS) may be limiting the metabolic flux toward the production of flavonoids and anthocyanins and 2) there may be mutations in the gene that encode dihydroflavonols (DHQ, DHK, DHM) in greenish-purple, yellow, and green genotyes. The genus Nicotiana uses both DHQ and DHM as substrates (Polashock et al., 2002). The tobacco used in this study found to contain cyanidin-3-glucoside suggesting the utilization of only DHQ as substrates. The transgenic tobacco plants ectopically expressing $P d-C H S, P d-D F R, P d-A N S$, and $P d-U F G T 2$ contain only cyanidin derivatives suggesting the utilization of DHQ as in control plants. Nevertheless, the amount of anthocyanins (cyanidin derivatives) in these four transgenic types helps to determine the control point of anthocyanin metabolic flux and the function of the CHS, DFR, ANS, and UFGT in the anthocyanin metabolic pathway of european plum.

\section{Conclusions}

Compared with the purple european plum, the expression levels of $P d-C H S$ in the greenish-purple, yellow, and green genotypes was severely downregulated. Our results suggest that CHS may be a limiting step in the production of anthocyanins in the european plum. Among the two copies of genes for UDP-glucose:flavonoid 3-O-glucosyltransferase, $P d$-UFGT 1 expression levels were high in yellow 2 and green genotypes, suggesting that flavonols and flavones may be the key substrates for UFGT 1 . Just as $P d-C H S$, the expression levels of $P d-U F G T 2$ were also severely downregulated in greenish-purple, yellow and green genotypes, suggesting that anthocyanidins may be the preferred substrates for UFGT 2 . The contents of individual polyphenols and the anthocyanins in green and yellow genotypes were considerably low compared with the purple genotype. More importantly, delphinidin derivatives were significantly low in green genotypes compared with yellow, greenish-purple and purple genotypes. This suggests that flavonoid substrate specificity (quercetin, kaempferol, myricetin) for DFR enzyme and metabolic flux for anthocyanin derivatives (pelargonidin, cyanidin, delphinidin) differs in green genotypes compared with yellow, greenish-purple, purple genotypes resulting in different colored european plum. 


\section{Literature Cited}

Abdi, N., W.B. McGlasson, P. Holford, M. Williams, and Y. Mizrahi. 1998. Responses of climacteric and suppressed-climacteric plums to treatment with propylene and 1-methylcyclopropene. Postharvest Biol. Technol. 14:29-39.

Boss, P.K., C. Davies, and S.P. Robinson. 1996. Analysis of the expression of anthocyanin pathway genes in developing Vitis vinifera $\mathrm{L}$. cv shiraz grape berries and the implications for pathway regulations. J. Plant Physiol. 111:1059-1066.

Camara, B., P. Hugueney, F. Bouvier, M. Kuntz, and R. Moneger. 1995. Biochemistry and molecular biology of chromoplast development. Intl. Rev. Cytol. 163:175-247.

Davies, K.M. and K.E. Schwinn. 2004. Transcriptional regulation of secondary metabolism. Funct. Plant Biol. 30:913-925.

Dooner, H.K., T.P. Robbins, and R.A. Jorgensen. 1991. Genetic and developmental control of anthocyanin biosynthesis. Annu. Rev. Genet. 25:173-199.

Durbin, M.L., B. McCaig, and M.T. Clegg. 2000. Molecular evolution of the chalcone synthase multigene family in the morning glory genome. Plant Mol. Biol. 42:79-92.

Espana, L., J.A. Heredia-Guerrero, J.J. Reina-Pinto, R. FernandezMunoz, A. Heredia, and E. Dominguez. 2014. Transient silencing of chalcone synthase during fruit ripening modifies tomato epidermal cells and cuticle properties. Plant Physiol. 166:1371-1386.

Fournand, D., A. Vicens, L. Sidhoum, J. Souquet, M. Moutounet, and V. Cheynier. 2006. Accumulation and extractability of grape skin tannins and anthocyanins at different advanced physiological stages. J. Agr. Food Chem. 54:7331-7338.

Gould, K.S., D.A. Dudle, and H.S. Neufeld. 2010. Why some stems are red: Cauline anthocyanins shield photosystem II against high light stress. J. Expt. Bot. 61:2707-2717.

Hellens, R.P., E.A. Edwards, N.R. Leyland, S. Bean, and P.M. Mullineaux. 2000. pGreen: a versatile and flexible binary Ti vector for Agrobacterium-mediated plant transformation. Plant Mol. Biol. 42:819-832.

Hily, J., R. Scorza, K. Webb, and M. Ravelonandro. 2005. Accumulation of the long class of siRNA is associated with resistance to plum pox virus in a transgenic woody perennial plum tree. Mol. Plant Microbe Interact. 18:794-799.

Holton, T.A. and E.C. Cornish. 1995. Genetics and biochemistry of anthocyanin biosynthesis. Plant Cell 7:1071-1083.

Honda, C., N. Kotoda, M. Wada, S. Kondo, S. Kobayashi, J. Soejima, Z. Zhang, T. Tsuda, and T. Moriguchi. 2002. Anthocyanin biosynthetic genes are coordinately expressed during red coloration in apple skin. Plant Physiol. Biochem. 40:955-962.

Horsch, R.B., S.G. Rogers, and R.T. Fraley. 1985. Transgenic plants, p. 433-437. Cold Spring Harbor Symp. Quantitative Biol. Vol. 50. Cold Spring Harbor Laboratory Press, Cold Spring Harbor, NY.

Jaakola, L., K. Maatta, A.M. Pirttila, R. Torronen, S. Karenlampi, and A. Hohtola. 2002. Expression of genes involved in anthocyanin biosynthesis in relation to anthocyanin, proanthocyanidin, and flavonol levels during bilberry fruit development. Plant Physiol. 130:729-739.

Jiao, Y., R.J. Ma, Z.J. Shen, J. Yan, and M.L. Yu. 2014. Gene regulation of anthocyanin biosynthesis in two blood-flesh peach (Prunus persica (L.) Batsch) cultivars during fruit development. J. Zhejiang Univ. Sci. 15:809-819.

Jones, D.H. 1984. Phenylalanine ammonia-lyase: Regulation of its induction, and its role in plant development. Phytochemistry 23:1349-1359.

Kahlau, S. and R. Bock. 2008. Plastid transcriptomics and translatomics of tomato fruit development and chloroplast-to-chromoplast differentiation: Chromoplast gene expression largely serves the production of a single protein. Plant Cell 20:856-874.

Kim, S.H., J.R. Lee, S.T. Hong, Y.K. Yoo, G. An, and S.R. Kim. 2003. Molecular cloning and analysis of anthocyanin biosynthesis genes preferentially expressed in apple skin. Plant Sci. 165:403-413.
Kobayashi, S., N. Goto-Yamamoto, and H. Hirochika. 2004. Retrotransposon-induced mutations in grape skin color. Science 304:982.

Kotepong, P., S. Ketsa, and W.G. van Doorn. 2011. A white mutant of malay apple fruit (Syzygium malaccense) lacks transcript expression and activity for the last enzyme of anthocyanin synthesis, and the normal expression of a MYB transcription factor. Funct. Plant Biol. 38:75-86.

Lim, E.K., S. Baldauf, Y. Li, L. Elias, D. Worrall, S.P. Spencer, R.G. Jackson, G. Taguchi, J. Ross, and D.J. Bowles. 2003. Evolution of substrate recognition across a multigene family of glycosyltransferases in Arabidopsis. Glycobiology 13:139-145.

Lister, C.E., J.E. Lancaster, and J.R. Walker. 1996. Phenylalanine ammonia-lyase (PAL) activity and its relationship to anthocyanin and flavonoid levels in New Zealand-grown apple cultivars. J. Amer. Soc. Hort. Sci. 121:281-285.

Li, X.J., J.Q. Zhang, Z.C. Wu, B. Lai, X.M. Huang, Y.H. Qin, H.C. Wang, and G.B. Hu. 2015. Functional characterization of a glucosyltransferase gene, LcUFGT1, involved in the formation of cyanidin glucoside in the pericarp of Litchi chinensis. Physiol. Plant. 156:139-149.

Meisel, L., B. Fonseca, S. González, R. Baeza-Yates, V. Cambiazo, R. Campos, M. González, A. Orellana, J. Retamales, and H. Silva. 2005. A rapid and efficient method for purifying high quality total RNA from peaches (Prunus persica L.) for functional genomics analyses. Biol. Res. 38:83-88.

Nakatsuka, T., Y. Abe, Y. Kakizaki, S. Yamamura, and M. Nishihara. 2007. Production of red-flowered plants by genetic engineering of multiple flavonoid biosynthetic genes. Plant Cell Rpt. 26:1951-1959.

Polashock, J.J., R.J. Griesbach, R.F. Sullivan, and N. Vorsa. 2002. Cloning of a cDNA encoding the cranberry dihydroflavonol-4reductase (DFR) and expression in transgenic tobacco. Plant Sci. 163:241-251.

Sui, X., X. Gao, M. Ao, Q. Wang, D. Yang, M. Wang, Y. Fu, and L. Wang. 2011. cDNA cloning and characterization of UDP-glucose: Anthocyanidin 3-O-glucosyltransferase in Freesia hybrida. Plant Cell Rpt. 30:1209-1218.

Szankowski, I., H. Flachowsky, H. Li, H. Halbwirth, D. Treutter, I. Regos, M. Hanke, K. Stich, and T.C. Fischer. 2009. Shift in polyphenol profile and sublethal phenotype caused by silencing of anthocyanidin synthase in apple (Malus sp.). Planta 229:681-692.

Takos, A.M., S.P. Robinson, and A.R. Walker. 2006. Transcriptional regulation of the flavonoid pathway in the skin of dark-grown 'Cripps' red apples in response to sunlight. J. Hort. Sci. Biotechnol. 81:735-744

Tzulker, R., I. Glazer, I. Bar-Ilan, D. Holland, M. Aviram, and R. Amir. 2007. Antioxidant activity, polyphenol content, and related compounds in different fruit juices and homogenates prepared from 29 different pomegranate accessions. J. Agr. Food Chem. 55:9559-9570.

Untergasser, A., H. Nijveen, X. Rao, T. Bisseling, R. Geurts, and J.A. Leunissen. 2007. Primer3Plus, an enhanced web interface to Primer3. Nucleic Acids Res. 35:W71-W74.

Wang, Z., D. Meng, A. Wang, T. Li, S. Jiang, P. Cong, and T. Li. 2013. The methylation of the PcMYB10 promoter is associated with greenskinned sport in Max Red Bartlett pear. Plant Physiol. 162:885-896. Waterhouse, A. 2002. Folin-Ciocalteau micro method for total phenol in wine. Curr. Protocols Food Anal. Chem. 1.1.1-1.1.8.

$\mathrm{Xu}$, W., H. Peng, T. Yang, B. Whitaker, L. Huang, J. Sun, and P. Chen. 2014. Effect of calcium on strawberry fruit flavonoid pathway gene expression and anthocyanin accumulation. Plant Physiol. Biochem. 82:289-298.

Yang, Y., G. Yao, D. Zheng, S. Zhang, C. Wang, M. Zhang, and J. Wu. 2015. Expression differences of anthocyanin biosynthesis genes reveal regulation patterns for red pear coloration. Plant Cell Rpt. 34:189-198.

Zepeda, L., A. Reznickova, and L. Lohr. 2014. Overcoming challenges to effectiveness of mobile markets in US food deserts. Appetite 79:58-67.

Zhang, X. and C.J. Liu. 2015. Multifaceted regulations of gateway enzyme phenylalanine ammonia-lyase in the biosynthesis of phenylpropanoids. Mol. Plant 8:17-27. 
Supplemental Table 1. Primers used for gene expression analysis of anthocyanin biosynthetic genes in european plum genotypes.

\begin{tabular}{|c|c|}
\hline Primer name & Primer sequence \\
\hline$\overline{P d-P A L-\mathrm{F}}$ & 5-'GGGTCATGGACAGCATGAG-3' \\
\hline$P d-C H S-F$ & 5-'TGTGTTGATCAGGCCACCT-3' \\
\hline$P d-D F R-F$ & 5-'GAGACGCACTTGACGCTGT-3' \\
\hline$P d-D F R-\mathrm{R}$ & 5-'GTGGCGACATGGAACACTC-3' \\
\hline$P d-A N S-\mathrm{F}$ & 5-'GGCCTCAAGTTCCAACCAT-3' \\
\hline$P d-U F G T 1-\mathrm{R}$ & 5-'TGGGCTTAGGGTTTTGAGG-3' \\
\hline$P p-U F G T 2-\mathrm{F}$ & 5-'CGCCTCACCTGCTTGATTA-3' \\
\hline$P p-U F G T 2-\mathrm{R}$ & 5-'AAGTCCGGAGAGCCAAAGA-3' \\
\hline Ps-Actin-F & 5-'CATGCCATTCTTCGTCTGG-3' \\
\hline Ps-Actin-R & 5-'TCAGCAGTGGTGGTGAACA-3' \\
\hline
\end{tabular}

Supplemental Table 2. Accession numbers of anthocyanin biosynthetic genes from european plum.

\begin{tabular}{lr}
\hline Gene name & Accession no. \\
\hline$P d$-PAL 1 & KJ461724 \\
$P d$-PAL 2 & KJ461725 \\
$P d$-CHS & KJ461726 \\
$P d$-DFR & KJ461727 \\
$P d$-ANS & KJ461728 \\
$P d$-UFGT 1 & KJ461729 \\
$P d$-UFGT 2 & JX149550 \\
$P s$-Actin & EF585293 \\
\hline
\end{tabular}

Supplemental Table 3. Primers used for the isolation of anthocyanin biosynthetic genes from european plum.

\begin{tabular}{|c|c|}
\hline Primer name & Primer sequence \\
\hline$P d-P A L-\mathrm{F}$ & 5'-ATCTGGGTTTTTGGTAATTAAC-3' \\
\hline$P d-P A L-\mathrm{R}$ & 5' -GAAGTGGTAAAGAACTTAGAAAGAA-3' \\
\hline$P d-C H S-\mathrm{F}$ & 5'-ACCTTTCTTGACGATCGGAGAAA-3' \\
\hline$P d-D F R-F$ & 5'-CAGTACATACTTGATTTGATTCAAAT-3' \\
\hline$P d-D F R-\mathrm{R}$ & 5' -AATATATAGCCAGATTGGATCATAGTTA-3' \\
\hline$P d-A N S-\mathrm{F}$ & 5'-AAGAGTTCAGAGCTTACGAAGAG-3' \\
\hline$P d-U F G T 1-\mathrm{F}$ & 5' -AACAAGTCAAAGGAAAACAAAAT-3' \\
\hline$P d-U F G T 1-\mathrm{R}$ & 5'-TGAAGACTCAACGTACGGAC-3' \\
\hline
\end{tabular}


Supplemental Table 4. Primers used for the genetic transformation of anthocyanin biosynthetic genes (ABG) and to examine the presence of ABG in transgenic tobacco plants.

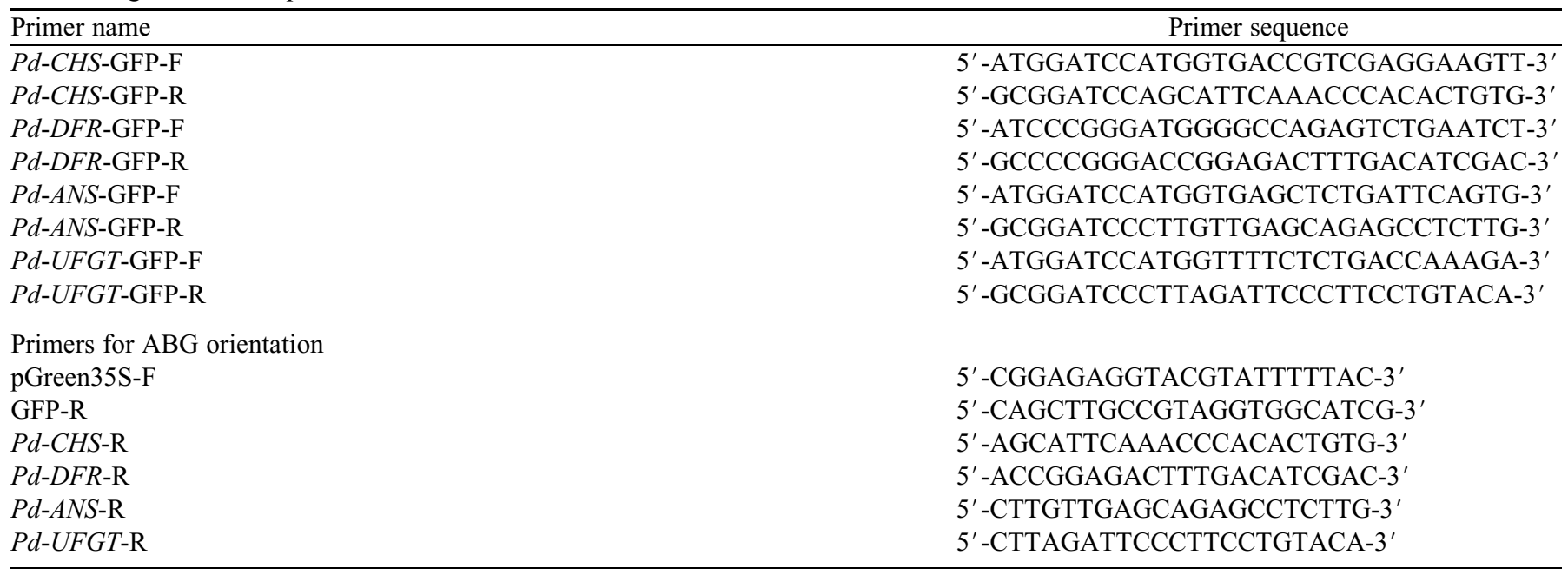

Supplemental Table 5. Total polyphenol content of different colored cultivars of european plum eluted in $100 \%$ methanol fraction.

\begin{tabular}{lc}
\hline Genotype & Total polyphenols (mg/100 g malic acid equivalent fresh weight \pm SD) \\
\hline Purple (V982017) & $1,334 \pm 32.4 \mathrm{a}^{\mathrm{z}}$ \\
Greenish purple (V90271) & $956 \pm 31.7 \mathrm{c}$ \\
Yellow 1 (V91057) & $1,231 \pm 40.1 \mathrm{~b}$ \\
Yellow 2 (V91058) & $1,249 \pm 49.6 \mathrm{ab}$ \\
Green 1 (V91048) & $439 \pm 34.6 \mathrm{~d}$ \\
Green 2 (V95141) & $329 \pm 21.0 \mathrm{e}$
\end{tabular}

${ }^{\text {z}}$ Samples with shared letters are not significantly different at $P<0.05$ using Tukey's multiple means comparison test.

Supplemental Table 6. Liquid chromatography-mass spectrometry profile of polyphenols from the european plum purple genotype V982017.

\begin{tabular}{|c|c|c|}
\hline Compounds & Mass fragments ions $(\mathrm{m} / \mathrm{z})^{\mathrm{z}}$ & Relative peak area (per $100 \mathrm{~g}$ fresh weight $\pm \mathrm{sD}$ ) \\
\hline Phenolic acids & & $19.77 \pm 0.6 \mathrm{a}^{\mathrm{y}}$ \\
\hline Quinic acid & $191.1,193$ & \\
\hline Caffeic acid glucoside & 341.2 & \\
\hline Courmaroyl quinate & 337.2 & \\
\hline Feruloyl quinate & 367.2 & \\
\hline Diferulic acid & 385.3 & \\
\hline \multicolumn{3}{|l|}{ Flavanones } \\
\hline Dihydrochalcone & 721.2 & \\
\hline Quinochalcone & $593.2,613.3$ & \\
\hline Flavones & $487.4,383.2$ & \\
\hline \multicolumn{3}{|l|}{ Flavonols } \\
\hline Kaempferol & $497.4,533.2,707.2,823.7,289.1$ & \\
\hline Delphinidin-3-0-G & $611.1,627.4$ & $42.4 \pm 0.9 \mathrm{a}$ \\
\hline
\end{tabular}

${ }^{\mathrm{z}}$ Ions having a specific mass-to-charge ratio.

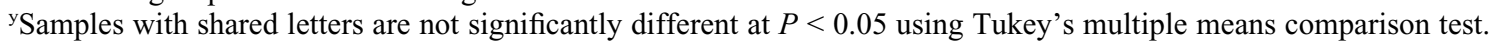


Supplemental Table 7. Liquid chromatography-mass spectrometry profile of polyphenols from the european plum greenish-purple genotype V90271.

\begin{tabular}{lcc}
\hline Compounds & Mass fragments ions $(\mathrm{m} / \mathrm{z})^{\mathrm{z}}$ & Relative peak area (per 100 g fresh weight $\pm \mathrm{SD})$ \\
\hline Phenolic acids & 133.1 & $18.04 \pm 0.6 \mathrm{c}^{\mathrm{y}}$ \\
Malic acid & 191.1 & \\
Quinic acid & 353.2 & \\
Chlorogenic acid & 385.3 & $18.04 \pm 0.3 \mathrm{e}$ \\
Diferulic acid & 197.1 & \\
Dihydroferulic acid & 313.2 & \\
Caffeoyl tartaric acid & & $63.91 \pm 2.1 \mathrm{~d}$ \\
Flavonoid aglycones & & $16.07 \pm 1.8 \mathrm{~b}$ \\
Flavonols & $445.4,497.4,533.2,661.5,707.2$ & $0.44 \pm 0.0 \mathrm{c}$ \\
Kaempferol & 949.8 & $45.76 \pm 0.6 \mathrm{~b}$ \\
Quercetin & & \\
Anthocyanins & $475.1,595.1,637.2,679.5$ & \\
Pelagonidin-3-0-G & $773.3,435.3,611.1,627.4$, & \\
Cyanidin-3-0-G & $799.2,977.2$ & \\
Delphinidin-3-0-G & & \\
Malvidin 3-0-G & & \\
\hline
\end{tabular}

${ }^{\mathrm{z}}$ Ions having a specific mass-to-charge ratio.

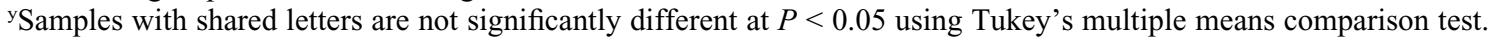

Supplemental Table 8. Liquid chromatography-mass spectrometry profile of polyphenols from the european plum yellow 1 genotype V91058.

\begin{tabular}{lcr}
\hline Compounds & Mass fragments ions $(\mathrm{m} / \mathrm{z})^{\mathrm{z}}$ & Relative peak area (per 100 g fresh weight $\pm \mathrm{sD})$ \\
\hline Phenolic acids & 191.1 & $22.6 \pm 0.2 \mathrm{~b}^{\mathrm{y}}$ \\
Quinic acid & 353.2 & $22.0 \pm 0.5 \mathrm{~b}$ \\
Chlorogenic acid & 385.3 & \\
$\quad$ Diferulic acid & & \\
Flavonoid aglycones & $421.3,613.3$ & \\
Flavanones & 613.3 & $55.3 \pm 0.9 \mathrm{~b}$ \\
Chalcone & & $19.21 \pm 0.7 \mathrm{a}$ \\
Quinochalcone & $445.4,497.4,533.2,705.2,707.2,823.7$ & $1.0 \pm 0.0 \mathrm{c}$ \\
Flavonols & 679.5 & $34.97 \pm 0.9 \mathrm{~b}$ \\
Kaempferol & $927.6,773.3$ & \\
Anthocyanins & $435.3,611.1,627.4$ & \\
Pelagonidin-3-0-G & & \\
Cyanidin-3-0-G & & \\
Delphinidin-3-0-G & & \\
\hline
\end{tabular}

${ }^{\mathrm{z}}$ Ions having a specific mass-to-charge ratio.

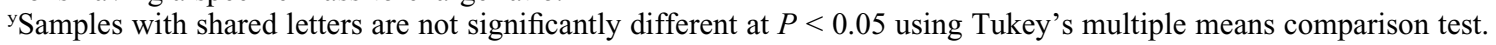

Supplemental Table 9. Liquid chromatography-mass spectrometry profile of polyphenols from the european plum yellow 2 genotype V91057.

\begin{tabular}{lcc}
\hline Compounds & Mass fragments ions $(\mathrm{m} / \mathrm{z})^{\mathrm{z}}$ & Relative peak area (per 100 g fresh weight $\pm \mathrm{sD})$ \\
\hline Phenolic acids & 191.1 & $24.7 \pm 0.9 \mathrm{~b}^{\mathrm{y}}$ \\
Quinic acid & 353.2 & $20.9 \pm 1.3 \mathrm{c}$ \\
Chlorogenic acid & 385.3 & \\
$\quad$ Diferulic acid & & \\
Flavonoid aglycones & $421.3,613.3$ & \\
Flavanones & & $54.3 \pm 0.5 \mathrm{c}$ \\
Quinochalcone & $445.4,497.4,533.2,705.2,707.2,823.7$ & $18.6 \pm 0.9 \mathrm{a}$ \\
Flavonols & & $0.7 \pm 0.01 \mathrm{c}$ \\
Kaempferol & 679.5 & $34.9 \pm 0.5 \mathrm{~b}$ \\
Anthocyanins & 773.3 & \\
Pelagonidin-3-0-G & $435.3,611.1,627.4$ & \\
Cyanidin-3-0-G & &
\end{tabular}

${ }^{\mathrm{z}}$ Ions having a specific mass-to-charge ratio.

${ }^{\text {y }}$ Samples with shared letters are not significantly different at $P<0.05$ using Tukey's multiple means comparison test. 
Supplemental Table 10. Liquid chromatography-mass spectrometry profile of polyphenols from the european plum green 1 genotype V91048.

\begin{tabular}{lcc}
\hline Compounds & Mass fragments ions $(\mathrm{m} / \mathrm{z})^{\mathrm{z}}$ & Relative peak area (per $100 \mathrm{~g}$ fresh weight $\pm \mathrm{SD})$ \\
\hline Phenolic acids & & $30.9 \pm 1.6 \mathrm{c}^{\mathrm{y}}$ \\
Quinic acid & 341.1 & \\
Caffeic acid glucoside & 353.2 & $34.8 \pm 1.0 \mathrm{~d}$ \\
Chlorogenic acid & 359.2 & \\
Dihydroferulic acid glucoside & 385.3 & \\
Diferulic acid & & \\
Flavonoid aglycones & & \\
Flavonols & $497.4,533.2,707.2$ & \\
Kaempferol & 289.1 & $0.5 \pm 0.02 \mathrm{a}$ \\
Kaempferide & 439.2 & $33.7 \pm 1.4 \mathrm{e}$ \\
Quercetin & & $28.1 \pm 1.7 \mathrm{a}$ \\
Anthocyanidin alycones & & $4.8 \pm 0.02 \mathrm{~b}$ \\
Monardaein, pelargonidin & 679.5 & $0.7 \pm 0.02 \mathrm{c}$ \\
Anthocyanins & 912.2 & \\
Pelagonidin-3-0-G & $611.1,773.3$ & \\
Cyanidin-3-0-G & $627.4,821.1$ & \\
Delphinidin-3-0-G & & \\
Malvidin-3-0-G & & \\
\hline
\end{tabular}

${ }^{\mathrm{z}}$ Ions having a specific mass-to-charge ratio.

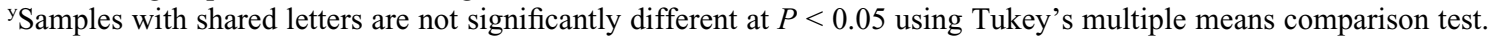

Supplemental Table 11. Liquid chromatography-mass spectrometry profile of polyphenols from the european plum green 2 genotye V91057.

\begin{tabular}{|c|c|c|}
\hline Compounds & Mass fragments ions $(\mathrm{m} / \mathrm{z})^{\mathrm{z}}$ & Relative peak area (per $100 \mathrm{~g}$ fresh weight $\pm \mathrm{sD}$ ) \\
\hline Phenolic acids & & $27.0 \pm 0.6 \mathrm{~d}^{\mathrm{y}}$ \\
\hline Quinic acid & 191.1 & \\
\hline Chlorogenic acid & 353.2 & \\
\hline Diferulic acid & 385.3 & \\
\hline Dihydriferulic acid & 197.1 & \\
\hline Coumaric acid & 163.1 & \\
\hline Feruloyl quinate & 367.3 & \\
\hline Hydroxybenzoic acid & 139.2 & \\
\hline Flavonoid aglycones & & $27.8 \pm 1.8 \mathrm{f}$ \\
\hline Flavanones & & \\
\hline Isoflavonoid & $39.2,671.3,421.3$ & \\
\hline Anthocyanins & & $56.7 \pm 1.3 \mathrm{f}$ \\
\hline Pelagonidin-3-0-G & $475.1,637.2,679.5$ & $44.3 \pm 1.4 \mathrm{a}$ \\
\hline Cyanidin-3-0-G & 611.1 & $10.5 \pm 0.06 \mathrm{~b}$ \\
\hline Delphinidin-3-0-G & $627.4,799.2$ & $1.8 \pm 0.1 \mathrm{c}$ \\
\hline
\end{tabular}

${ }^{\mathrm{z}}$ Ions having a specific mass-to-charge ratio.

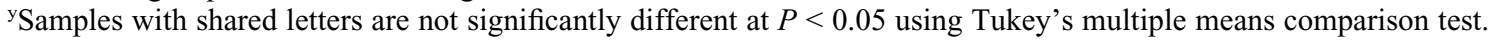

